Restricted effects on cancer, as noted by Kontis and colleagues, ${ }^{1}$ partly shows the long latency of this disease and relative delays in benefits of some interventions. A long-term perspective is needed for vaccines against human papilloma viruses and hepatitis B virus, both part of the wider GMF indicators. For these examples, immunisation of adolescents and infants respectively, will only show benefits two to three decades after the 2025 target date. However, this should not be a reason against immediate implementation of these vaccines or other highly effective strategies for cancer prevention.

Another reason for the slight effect on cancer mortality is because of the greater heterogeneity in its risk factors than for other NCDs. Of the six factors accounted for by Kontis and colleagues, ${ }^{1}$ only tobacco, alcohol, and obesity are a risk for cancer. The greater variability in causes of cancer should be translated into a more nuanced approach for control of this disease, including tailored responses at a national and regional level. ${ }^{3}$ For example, chronic infections are associated with one in six cancers globally, one in three in sub-Saharan Africa, but only one in thirty in Australasia. ${ }^{4}$ Therefore, the inclusion of the two previously mentioned vaccines, together with cervical cancer screening in WHO's indicators for NCDs is positive. However, additional cancer risk factors include exposure to excess sunlight, air pollution, various occupational exposures, and many naturally occurring or industrial chemicals, which are all potential targets for primary prevention. ${ }^{5}$

Breast cancer represents a different problem. In women of the target age range for GMF (30-69 years), breast cancer is the most common form of cancer deaths in all world regions and represents one in five of all female cancer diagnoses. ${ }^{6}$
Overweight and obesity are associated with post-menopausal risk of breast cancer, but the so-called halting the rise scenario for obesity, reported by Kontis and colleagues, ${ }^{1}$ can do little to counterbalance the continuing rise of the disease in low-income and middle-income countries because of women having their first child at an older age and a fewer number of children. Of ten lead NCDs in causes of mortality tabulated by Kontis and colleagues, breast cancer is the only disease for which no net mortality reduction would happen even if the $25 \times 25$ risk factor targets were achieved in full. Nevertheless, breast cancer deaths can be averted, with a high rate of success, by policies of early detection and effective treatment with multi-modality therapy. This rate of success can also be achieved in developing countries if availability and access to treatment are improved by appropriate investments in health services. For prostate cancer, the most common incident cancer in men worldwide, the situation is even more challenging because of an absence of identified risk factors precluding primary prevention, while present screening approaches fall short of requirements.

The heightened focus on tackling NCDs is a landmark step in global public health. National cancer control certainly needs to embrace the benefits of addressing common, modifiable NCD risk factors, and respond to the absence of investments in health service infrastructure. However, equally imperative is for governments to look beyond the control of NCDs to develop integrated, cancer control plans that can be adapted to the local cancer profile. Caution is needed to ensure that the merited focus on certain NCDs, brought about by the $25 \times 25$ target, does not become a constraint in reduction of the burden from others.
We declare no competing interests.

*Christopher P Wild, Freddie Bray,

David Forman, Silvia Franceschi,

Rengaswamy Sankaranarayanan,

Kurt Straif

director@iarc.fr

International Agency for Research on Cancer Lyon 69372, France

1 Kontis V, Mathers CD, Rehm J, et al. Contribution of six risk factors to achieving the $25 \times 25$ non-communicable disease mortality reduction target: a modelling study. Lancet 2014 384: 427-37.

2 WHO. Draft comprehensive global monitoring framework and targets for the prevention and control of noncommunicable diseases. Geneva; World Health Organization, 2013. http://apps.who.int/gb/ebwha/pdf_ files/WHA66/A66_8-en.pdf?ua=1 (accessed Oct 2, 2014).

3 Wild CP. The role of cancer research in noncommunicable disease control. J Natl Cancer Inst 2012; 104: 1051-58.

4 de Martel C, Ferlay J, Franceschi S, et al. Global burden of cancers attributable to infections in 2008: a review and synthetic analysis. Lancet Oncol 2012; 13: 607-15.

5 Vineis P, Wild CP. Global cancer patterns: causes and prevention. Lancet 2014 383: 549-57.

6 Ferlay J, Soerjomataram I, Ervik M, et al. GLOBOCAN 2012 v1.0, cancer incidence and mortality worldwide, IARC CancerBase No. 11. Lyon, France: International ARC; 2013. http:// globocan.iarc.fr (accessed Oct 2, 2014).

\section{Challenges for Colombian medical journals}

Jun-Song Yang and Ding-Jun Hao reported in their Correspondence (Aug 30, p 743) $)^{1}$ that Chinese medical journals are encountering an unprecedented crisis related to a general upward tendency of Chinese papers to be published in leading international journals. We believe that this situation is not specific to China, but also concerns other countries, such as Colombia.

Until 2013, journals in Colombia (national and international) were classified in four categories A1, A2, $B$, and $C$. However, the classification of scientific journals (medical and non-medical) changed in 2013 to increase the quality of submissions to national journals. Since the beginning 


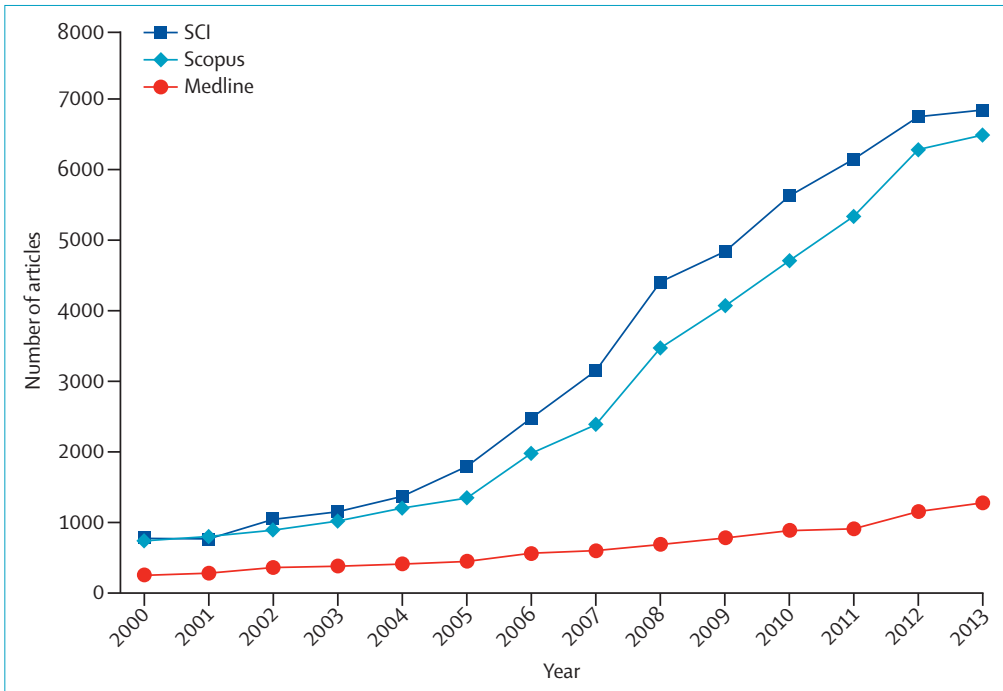

Figure: Number of articles from Colombia listed in Science Citation Index (SCI), Scopus, and Medline between 2000 and 2013

of 2014, journals in Colombia are classified according to citation indicators (into six categories A1-A4, $B$, and $C$ ) derived from the Science Citation Index $(\mathrm{SCl})$ and Scopus, including the Impact Factor and the SCImago Journal Rank, in quartiles of Q1 to Q4 which are equivalent to the previous Colombian classification $(A 1, A 2, B$, and $C)$. In the new classification, journals in $B$ and $C$ categories correspond with journals indexed in other international and regional databases (eg, SciELO, LILACS, and Redalyc).

The number of Colombian articles listed in SCl, Scopus, and Medline has rapidly increased since mid-2000s (figure). In Colombia, an article published in a journal of a high category is awarded a bonus. Many researchers published in Colombian national journals classified in the top category (A1) because international journals with high impact factors would also be classified in A1 or a lower category. ${ }^{2}$

Conversely to events up to 2013 (whereby a journal with a high impact factor was classified as A2 or B by the Publindex from the Colombian Science Department), ${ }^{2}$ Colombian journals now aspire to be classed as A1 from Publindex and Q1 category at $\mathrm{SCl}$ or Scopus. This change represents a complex challenge for many Colombian journals that will now have to increase their quality to remain in a high classification, ${ }^{3}$ otherwise, as many do, they will fall into lower categories.

Data by SCI and Scopus from 2013 show that no Colombian journals are in the Q1 category, and therefore, with implementation of new classification criteria no Colombian journals will be listed in A1. Only international journals at Q1 will be classified in Colombia as A1. Few Colombian journals were Q2 (equivalent to $A 2$ ) and most of these were included as Q3 (A3) and Q4 (A4) categories.

Colombian journals publish their content in Spanish and English to increase their visibility and impact factor in the international scientific community, ${ }^{4}$ but also to maintain their local role. Additional strategies can be developed, but implementation of unethical practices, such as citation stacking, should not be used. ${ }^{5}$ How Colombian journals assume this challenge will be seen in

AJRM is associated editor of Infectio (journal of the Colombian Association for Infectious Diseases) and assistant editor of Revista Medica de Risaralda (RMR) (Pereira, Colombia). PMT is associated editor of RMR. We declare no competing interests. the near future.
*Alfonso J Rodríguez-Morales, Percy Mayta-Tristán arodriguezm@utp.edu.co

Public Health and Infection Group of Research, Faculty of Health Sciences, Universidad Tecnológica de Pereira, Pereira, Risaralda, Colombia (AJRM); and Universidad Peruana de Ciencias Aplicadas, Lima, Perú (PMT)

1 Yang J-S, Hao D-J. The crisis of Chinese medical journals. Lancet 2014; 384: 743-44.

2 Rodriguez-Morales AJ, Ochoa-Orozco SA, Mayta-Tristán P. Impact of Colombian health journals: comparing Publindex versus Google Scholar Metrics, SciELO and SCOPUS. Rev Cuba Inf Cienc Salud 2014; 25: 24-35.

3 Rodríguez-Morales AJ. Infectio and the new policies of Publindex. Infectio 2014; 18: 01-02.

4 González-Alcaide G, Valderrama-Zurián JC, Aleixandre-Benavent R. The impact factor in non-english-speaking countries. Scientometrics 2012; 92: 297-311.

5 Van Noorden R. Brazilian citation scheme outed. Nature 2013; 500: 510-11.

\section{Department of Error}

Glasier AF, Cameron ST, Fine PM, et al. Ulipristal acetate versus levonorgestrel for emergency contraception: a randomised non-inferiority trial and meta-analysis. Lancet 2010; 375: 555-62-In this Article (Jan 29, 2010), the number of pregnancies in the levonorgestrel group (0-24 h time window) reported in table 2 should be 14 instead of 15 and the corresponding pregnancy rate should be $2.3 \%$ instead of $2.5 \%$. This error does not change the odds ratio or $p$ values, which were calculated through an independent logistic regression model. The correction has been made to the online version as of Oct 24, 2014

Murray CJL, Ortblad KF, Guinovart C, et al. Global, regional, and national incidence and mortality for HIV, tuberculosis, and malaria during 1990-2013: a systematic analysis for the Global Burden of Disease Study 2013. Lancet 2014; 384: 1005-70-In the Summary of this Article (July 22, 2014), the third sentence of the Interpretation should have read "Incidence rates for HIV, tuberculosis, and malaria have all decreased since 2000." This correction has been made to the online version as of Oct 24, 2014. 